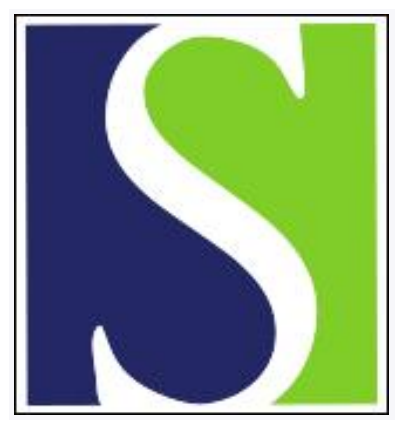

Scand J Work Environ Health 2010;36(2):121-133

https://doi.org/10.5271/sjweh.2900

Published online: 01 Feb 2010, Issue date: 00 Mar 2010

Shift work, sleep, and sleepiness - differences between shift schedules and systems

by Sallinen M, Kecklund $G$

Affiliation: Brain and Work Research Center, Finnish Institute of Occupational Health, 00250 Helsinki. mikael.sallinen@ttl.fi

Refers to the following texts of the Journal: 1998;24 suppl 3:69-75

1998;24 suppl 3:49-54 2008;34(3):198-205 2004;30(2):149-156

The following articles refer to this text: $2010 ; 36(2): 81-84$;

2015;41(3):268-279; 2015;41(4):413-416; 2017;43(6):569-577;

2018;44(4):394-402; 2020;46(2):161-167; 2020;46(4):446-453;

2020;46(5):516-524; 2020;46(6):570-578; 2020;46(6):557-569;

2021;47(5):395-403; 2021;47(6):446-455; 2022;48(7):507-510

Key terms: 2-shift work; 3-shift work; extended operation; health; health; irregular shift system; night work; review; shift schedule; shift system; shift work; sleep; sleep-wake pattern; sleepiness; workhours; working hours

This article in PubMed: www.ncbi.nlm.nih.gov/pubmed/20119631

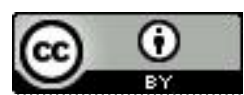




\title{
Shift work, sleep, and sleepiness - differences between shift schedules and systems
}

\author{
by Mikael Sallinen, PhD, ${ }^{1,2}$ Göran Kecklund, PhD ${ }^{3}$
}

Sallinen M, Kecklund G. Shift work, sleep, and sleepiness - differences between shift schedules and systems. Scand J Work Environ Health. 2010;36(2):121-133.

\begin{abstract}
In this narrative review, we examined what level of research evidence is available that shift workers' sleep-wake disturbances can be minimized through ergonomic shift scheduling. We classified the pertinent studies conducted on real shift workers in field conditions by the type of shift system and study design (ie, whether the shift systems were modified or not - "treatment" versus "no treatment"). The results of the observational studies in which no changes to the shift system were made (ie, no treatment) showed that, irrespective of the shift system, night and early-morning shifts and quick returns are associated with short sleep and increases in sleepiness. The same is true for very long shifts ( $>16$ hours) and extremely long weekly working hours ( $>55$ hours). For all categories of shift systems, there were a lack of controlled intervention studies, limiting the possibility to provide solution-focused recommendations for shift scheduling. Most of the controlled intervention studies had been conducted on workers under regular 3-shift systems. These studies suggested that a change from slowly backward-rotating shifts to rapidly forward-rotating shifts is advantageous for alertness and, to some degree, sleep. We also found that a change from an 8- to 12-hour shift system does not necessarily result in impairments in the sleep-wake pattern. The level of research evidence was affected by many of the studies' frequent methodological limitations in measuring sleep and sleepiness. In all, to have reliable and solution-focused recommendations for shift scheduling, methodologically sound controlled intervention studies are required in different categories of shift systems.
\end{abstract}

Key terms 2-shift work; 3-shift work; extended operation; irregular shift system; night work; review; sleep-wake pattern; work hours.

Shift work is an ambiguous term that refers to a wide range of work hour arrangements involving two or more teams (shifts) that differ in terms of the starting and finishing times of their work. In this review, our aim was to examine the relationship between the different kinds of schedules and systems of shift work and the sleep-wake patterns of workers.

Sleep is known to be important for health and functional capacity. A loss of sleep has been shown to result in disturbances in many basic bodily functions, such as glucose metabolism $(1,2)$, excretion of hormones $(3$, 4 ), and the functions of the autonomous nervous and immune systems $(2,5,6)$. In light of these findings, it is not surprising that short habitual sleep is associated with an increased risk of, for example, coronary heart disease (7). Sleep restriction has also been shown to be detrimental to alertness and cognitive functions, the basics of our mental functional capacity $(8-10)$. Such effects of sleep loss at least partly explain why disturbed sleep puts an individual at an increased risk of occupational accidents $(11,12)$.

There are a number of good reviews on shift work and sleep-wake patterns, concluding that night shifts (eg, $\geq 3$ hours of the shift between $24.00-06.00$ hours) and early-morning shifts (starting between 04.0007.00 hours) are particularly associated with disturbances in the sleep-wake pattern during and outside the shifts (13-15). Typical patterns are a 2-4-hour truncation of sleep before the early-morning shift and after the night shift (day sleep) and an increase in the incidence

1 Brain and Work Research Center, Finnish Institute of Occupational Health, Helsinki, Finland.

2 Agora Center, University of Jyväskylä, Jyväskylä, Finland.

3 Stress Research Institute, Stockholm University, Stockholm, Sweden.

Correspondence to: Mikael Sallinen, Brain and Work Research Center, Finnish Institute of Occupational Health, Topeliuksenkatu 41a A, 00250 Helsinki. [E-mail: mikael.sallinen@ttl.fi] 
of severe sleepiness and unintended sleep during shifts. The prevalence rates of severe sleepiness are around $50 \%$ and $25 \%$ in the night and early-morning shifts, respectively, while the rate is around $5 \%$ in the day and evening shift (16). The main reason for these patterns and differences is the daytime-oriented circadian rhythm of alertness that makes it difficult to sleep during the day and be alert at night.

However, it is not just the timing of an individual shift that plays a significant role in shift worker's sleep; there are also a number of other important shift system characteristics. The most important features include: (i) the direction and speed of shift rotation, (ii) the length of the recovery period in between two successive shifts, (iii) the distribution of rest days during the shift system, (iv) the length of shifts, (v) the number of consecutive work shifts, and (vi) the timing of change-over (17).

The main question of this narrative review is: "What level of research evidence is available for recommendations on how shift schedules should be designed in order to protect workers from sleep-wake disturbances?" Until now, there have been a few reviews on the relationship of the specific characteristics of shift schedules, such as shift rotation and length (18-21), to the sleep-wake pattern. Our review differs from these in two respects. First, we classified the cited studies by the type of the shift systems they examined (eg, regular 3-shift system), not by the characteristics of a shift schedule (eg, speed of shift rotation). This approach made it possible to look at each shift system as an entity on its own. Second, we placed an emphasis on intervention studies, as did the two most recent reviews $(19,20)$, but in addition to these, we reviewed observational studies that included no "treatment" (ie, rescheduling of the shift system). We opted to include observational studies because many of them used good indicators of sleep and sleepiness [eg, polysomnography, actigraphs (wrist-worn activity monitors), and repeated ratings of sleepiness across shifts] and carried out measurements across days of the shift cycle, which provide valuable knowledge on sleep-wake patterns within a given shift system. This also meant that we could obtain information on shift systems lacking in intervention studies.

We collected the studies through a literature search in PubMed. We excluded studies from our review that did not: (i) present the design of the shift system, (ii) examine adequate samples ( $>6$ subjects), (iii) relate the results to specific shifts or shift schedules; or (iv) involve an examination of real shift workers in field conditions. This last requirement aimed to increase the ecological validity of our review and the comparability of the studies included. We accepted the outcome measures of sleep and sleepiness based on either questionnaire, behavioral, or physiological data.

\section{Studies on regular 3-shift systems}

Most of the studies on the sleep-wake pattern in regular 3 -shift work have focused on comparisons between the shift types or cycles that differ in terms of direction and speed of rotation. These studies include both observational and intervention studies.

Two observational studies published in three papers were identified in which the sleep-wake patterns of workers on regular 3-shift system were electrophysiologically recorded (22-24). Tilley et al (22) conducted a study on industrial workers showing that their sleep was, on average, one hour shorter prior to early-morning shifts than before afternoon shifts. In association with night shifts, the difference in sleep length was even greater ( $>1.5$ hours) compared to afternoon shifts. Another study conducted on papermill workers focused on the occurrence of signs of sleepiness in electroencephalogram (EEG) during various shifts (23). The main finding was that the spectral content of the EEG showed elevated levels of sleepiness during the night compared to afternoon shifts. A second paper on the same study showed that $28 \%$ of the shift workers involuntarily dozed off during the night shift and slept during approximately 40 minutes of the shift (24).

In the observational questionnaire study of Cruz et al (25), comparisons were made between air-traffic controllers, all of whom were on rapidly backward-rotating shift systems but worked night and early-morning shifts at different frequencies. Those who had worked night shifts in the last six months reported more napping at work and falling asleep while driving home compared to those who had not worked night shifts. Having more early-morning shifts in a 2 -week schedule $(\geq 4$ versus $<4$ ) was associated with shorter sleep (6.1 versus 6.7 hours). In addition, working longer shifts ( 8 versus 9 hours) was associated with increased dozing off.

Two observational questionnaire studies compared two groups of 3-shift workers whose shifts rotated in the opposite direction $(26,27)$. In both studies, the forwardrotating shifts were superior to the backward-rotating ones with regard to sleep quality or quantity, but less so with respect to sleepiness. In the study of Tucker at al (26), forward rotation was more advantageous than backward rotation only on morning shift days (6.13 versus 5.55 hours of sleep), while the result was reversed on evening shift days ( 8.22 versus 8.88 hours). In addition, the observed differences between the various 3 -shift systems were present only in continuous but not discontinuous shift systems. This finding suggests that it is not just the direction of rotation, but also the presence or absence of quick returns (eg, a changeover from a night to an evening shift or from an evening to a morning shift in a backwardrotating regular 3 -shift system) that matters. 
The relationship of 3-shift systems to sleep and sleepiness has also been examined in non-randomized controlled intervention studies. Rosa et al (28) rescheduled the work shifts at a steel-rolling mill to start an hour later than previously (shift changeovers at 07.00, 15.00, and 22.00 hours under the new schedule). The shift schedule rotated slowly backward in sequences of four consecutive shifts of each type. Following the change in shift changeovers, the participants extended their sleep by 30 minutes on morning shifts $(6.2$ hours before the change), while no significant changes occurred for those on evening ( 7.2 hours) or night shift (5.8 hours). Sleep quality improved on morning shift days and worsened, to some extent, on night and evening shift days. Selfrated sleepiness decreased during the morning shift and elevated during the night shift. In a control worksite, where shift changeover times remained unchanged, no significant changes occurred in sleep or alertness. An interesting finding was also that older workers ( $\geq 40$ years) averaged 1.2 hours less sleep than their younger counterparts ( $<40$ years) in association with night shifts. In all, the study proposed that even slight changes in shift start and end times are of importance for sleep and sleepiness when they occur within a normal night sleep period.

Most of the non-randomized controlled intervention studies have focused on the direction and speed of shift rotation. Barton et al (29) studied changes in the wellbeing of 293 car manufacturing workers when they reversed the direction of rotation of their forward-rotating, discontinuous 3-shift system (weekends off). After the change, the workers reported more difficulties in falling asleep and getting up and impairments in sleep quality on evening shift days. A control group that maintained the backward-rotating system throughout the study did not report such changes. In association with the other shifts, no changes in sleep occurred. Similar to Tucker et al (28), the authors concluded that the direction of rotation may be less important for adaptation to a shift schedule than the presence of quick returns.

The questionnaire study of Knauth \& Hornberger (30) was among the first to focus on the change from a continuous slowly backward-rotating shift system to a continuous rapidly forward-rotating system, but the results were not very encouraging: no changes in sleep duration or disturbances were observed after the intervention. More recently, Härmä et al (31) examined the effects of a similar shift change on the sleep-wake patterns of maintenance workers. After the introduction of a very rapidly rotating shift schedule (consisting of one morning, evening, and night shift in a row), the amount of sleep decreased after the night shift (day sleep) among the workers aged $\geq 45$ years and before the morning shift among their younger counterparts. Sleep quality was improved particularly among the younger workers as indicated by a decrease in the fragmentation index calculated from the actigraphic data. The level of subjective sleepiness decreased and vigilance performance improved during the night shift especially in the older age group. In the control group that remained on the original slowly backward-rotating shift system, no corresponding changes in the sleep-wake pattern occurred.

Recently, Viitasalo et al (32) conducted a study including a control group and replicated the finding of the positive effect on self-reported alertness when switching from a slowly backward-rotating shift system to a very rapidly forward-rotating one, even though the self-assessed probability of falling asleep remained unchanged. However, sleepiness was not measured during the shifts, but retrospectively and without taking into account which of the shifts, if any, was worked on a given day. These methodological deficiencies make it difficult to interpret the results.

A very recent study by Karlson et al (33) showed that a change from a rapidly forward-rotating shift system to a slowly backward-rotating one improved self-reported sleep quality and fatigue among the workers of a manufacturing plant. A group of daytime workers, serving as the control group, showed no corresponding improvements. This finding is surprising, as the change in shift schedule was opposite to the shift change in the abovementioned Finnish studies. However, no shift-specific data were collected in association with the various shifts, which makes it difficult to interpret the findings. In addition, the use of daytime workers as a control group may not have been an optimal choice.

To summarize, the results show that the sleepwake pattern of a worker on a regular 3-shift system is afflicted by, in particular, the early-morning and the night shift, but the way in which these shifts are organized also plays a role. Most of studies' results favor rapidly forward-rotating shifts. However, in the discontinuous shift system (weekends off), the direction of rotation probably does not matter as much as in the continuous shift system. It is likely that the advantages of the rapidly forward-rotating shift systems over the slowly backward-rotating one are not based just on the direction and speed of rotation, but also on the absence of quick returns.

\section{Studies on irregular 3-shift systems}

In transport and some other sectors, it is common to have very irregular shift systems. In contrast to regular 3 -shift schedules, the start and finish times, shift lengths and rest periods between shifts vary a lot in an irregular shift system. Thus, irregular shift systems are much 
more unpredictable and possibly more difficult to cope with than regular ones. Hence, one may assume that very irregular shift systems result in more sleep-wake disturbances. To our knowledge, there is no intervention study that has explored how sleep length or quality and sleepiness are affected by the rescheduling of an irregular shift system, but all studies on this shift system fall into the category of observational studies.

An early study by Åkerstedt \& Gillberg (34) compared different occupations that work regular or irregular shift systems with respect to sleep and sleepiness. On the whole, the were some differences between occupational groups, but there were no findings to support that those working irregular shift systems (represented by train drivers) have worse sleep or more sleepiness than groups doing regular shift work (eg, industrial workers). Another study on railway workers showed that individuals who worked an irregular shift system reported poorer sleep quality than those on the regular 3-shift system (35). However, the cross-sectional design and lack of information on whether the groups differed with respect to other occupational factors (eg, work stress) suggest that one should be cautious about drawing conclusions on these studies.

It was not possible to categorize the irregular shift work studies with respect to the direction or speed of rotation. The reason for this is that most papers did not present detailed information on the shift system. However, some papers showed that backwards rotation, quick returns, early-morning shifts and relatively slow rotation are common $(16,36-38)$. Several studies have described the relationship of irregular shift systems, particularly among train and truck drivers, with sleep and sleepiness. A few studies have used polysomnographical recordings in naturalistic environments. Torsvall et al (39) found that day sleep (following night work) was slightly $<5$ hours among train drivers, which was approximately 3 hours shorter than their sleep after a day shift. Foret \& Lantin (40) and Kecklund et al (41) also reported similar findings in connection with early-morning work.

In another paper on train drivers, Torsvall \& Åkerstedt (42) demonstrated a clear increase in physiological (EEG-defined) sleepiness during the night shift with increased alpha and theta power density and higher occurrence of slow eye movements. More recently, Mitler et al (43) made a large-scale study on truck drivers where they compared four different shift systems, of which only one included daytime work. A major finding was the short length of drivers' average sleep - 4.78 hours - irrespective of the type of work schedule. The shortest sleep (3.83 hours per 24 hours across 5 work days) was observed among a group of drivers who had 13 hours of (almost) permanent night driving. The longest sleep -5.38 hours - was found among the daytime group who had a relatively late start time (09.00 hours). During daytime, the prevalence of video-recorded sleepiness events was low $(1.5 \%$ of the entire recording time) whereas the highest prevalence of sleepiness events $(11.6 \%)$ occurred among the permanent night-driving group. A majority (85\%) of the sleepiness events occurred during evening and nighttime. The individual differences were also noteworthy and only a small group ( $10 \%$ of the drivers) accounted for $54 \%$ of the sleepiness events.

Most of the studies have used sleep-wake diaries, sometimes together with actigraphs. Sallinen et al (44) compared different combinations of shifts for both train drivers and traffic controllers. They found that the length of sleep between two night shifts was 5.7 hours among the train drivers. Also a morning shift preceded by an evening shift (a quick return in a regular shift system) resulted in short sleep ( $\approx 5.5$ hours). Early morning shifts, irrespective of the shift that preceded it, always seemed to result in insufficient sleep (slightly $<6$ hours). The sleep-wake rhythm was often characterized by "split sleep" (ie, sleep divided in two episodes across the 24-hour day with one longer sleep episode and one nap episode). Napping was most common in those shift combinations where the main sleep episode was most curtailed (44). For example, naps were observed in two thirds of the shift combinations that included a night shift; an even higher occurrence was observed when the start time of the night shift was delayed. Roach and coworkers (37) also found that train drivers often experienced short sleep and that the length and timing of the break between work shifts were important determinants of sleep length. Thus a break that was $\leq 12$ hours resulted in 3.1-7.9 hours of sleep depending on when the break occurred. When the break was $\geq 24$ hours, the sleep length was never $<6.8$ hours. Ingre et al (38) demonstrated the strong effects of start time on earlymorning work in irregular shift systems. In order to obtain 8 hours of sleep, the shift should not start before 10.00 hours. A shift that starts around 06.00 hours will on average result in 6 hours of sleep. Really short sleep ( $\leq 5$ hours) was observed among train drivers who started before 05.00 hours.

Härmä et al (16) examined self-rated sleepiness [Karolinska sleepiness scale (KSS) ratings] and found that severe sleepiness (defined as KSS $\geq 7$ ) peaked at nighttime and was much lower in the afternoon shift and somewhere in between during the morning shifts. Short work shifts and long sleep decreased the risk for severe sleepiness. The prevalence of self-reported dozing off, a stronger indicator of severe sleepiness, showed a similar result although the prevalence was slightly lower compared to the KSS $\geq 7$ metric (16). In another paper, Sallinen et al (45) demonstrated that the variation in sleepiness between different night shifts was large, although it was difficult to establish a systematic pattern related to 
combinations of shifts. The authors also found that early start time of the morning shift increased sleepiness. A high prevalence of sleepiness during the morning shift was also demonstrated in a study by Ingre et al (46) in which train drivers were experimentally scheduled to make the same trip but with three different start times. This means that work characteristics were very similar between the experimental conditions. A large majority $(82 \%)$ of the drivers in the earliest shift (start time 05.49 hours) rated sleepiness severe (KSS $\geq 7$ ) at least once during the work shift. However, severe sleepiness was also relatively common towards the end of the late shift (09.49-22.00 hours).

To sum up, very irregular shift systems often result in sleep loss and split sleep (in particular when the break time is reduced) when the shifts starts early (before 07.00 hours) in the morning and when sleep occurs during the daytime. The level of severe sleepiness was high during night work, but also those working earlymorning shifts showed elevated levels. It is not possible to conclude whether irregular shift systems are worse than regular ones, although the general impression of the studies is that individuals having irregular work schedules may be at higher risk of developing cumulative sleep loss and excessive work-related sleepiness. In addition, the shortage of controlled intervention studies makes it difficult to provide recommendations on how irregular shift systems should be designed to reduce sleep loss and severe sleepiness.

\section{Studies on 2-shift systems}

Regular 2-shift systems are common in many occupations. One type of 2-shift system involves only morning and evening work. If the start time of the morning shift is not too early, and if the break time between shifts is sufficiently long, such a shift system would probably not cause any severe sleep-wake problems. However, another type of 2-shift system is based on 12-hour shifts, alternating between day and night work and representing compressed work hours. One advantage with a 12-hour, 2-shift system is the increase in free days; it is not unusual to have 3-4 days off/week. This increases the opportunity to obtain sufficient recovery sleep during days off, which should prevent accumulation of sleepiness due to several consecutive days of partial sleep loss. However, the extension of work days may cause high levels of sleepiness towards the end of the shift, and possibly reduced sleep due to insufficient break time between shifts.

Tucker et al (47-49) have published a series of observational questionnaire studies on 12-hour shifts. One study (47) compared sleep in an 8-hour shift system (rapidly forward-rotating shift system, with 7 consecutive work days) to a 12 -hour system (2-4 consecutive work days). The study also examined whether shift changeover time would modify the results. The 12 -hour shift system resulted in longer sleep (18-30-minute increase) with the exception of sleep following the afternoon shift which was 2 hours longer than the corresponding night sleep in the 12-hour shift system. An early-shift changeover time had deleterious effects on sleep duration (30-minute decrease) prior to day shifts but was beneficial for day sleep between night shifts (30-minute increase). There were few significant interactions (shift length $\times$ changeover time). In general, the effect of changeover time seemed to be slightly larger than that of shift duration. In another paper, Tucker et al also evaluated the retrospective ratings of alertness and found that, compared to the 8-hour shift, the 12-hour shift was associated with slightly lower sleepiness during the second half of the night shift and the first half of the day shift, but higher sleepiness during the second half of the day shift (48). In another study (49), they compared 12-hour shift systems that differed with respect to the distribution of rest days and shift changeover times (06.00 versus 07.00 hours). Two of the shift systems had four consecutive work shifts followed by two days off, whereas the other shift systems had two rest days incorporated in the middle of the shift sequence (following the first two 12-hour day shifts). The distribution of rest days had only a marginal impact on sleep duration and quality; however, sleepiness was slightly lower for the shift system with two 12-hour shifts. Again an early shift changeover time resulted in shorter sleep and somewhat higher sleepiness in connection with the dayshift, whereas day sleep between night shifts was slightly longer. Thus, the effects of changeover time were in agreement with previous studies on regular 3-shift work (eg, 28).

Bednick \& coworkers (50) conducted a within-subject questionnaire study where they compared 12-hour day and night shifts, respectively. They found that the extended day shift was associated with less sleep (5.6 hours) than the extended night shift $(\approx 6$ hours depending on the shift). The short sleep in connection with the day shift was probably related to the early changeover time (06.00 hours). However, sleepiness was higher during the night. A recent study examined a shift system that had 12-hour shifts for 5-7 consecutive work days (51). There was a high prevalence $(61 \%)$ of severe sleepiness (KSS $\geq 7$ ) among 12-hour night shift workers compared to regular day (9\%) and 12-hour day shift workers (23\%). On the other hand, the prevalence of severe sleepiness during the 12-hour night shifts was not higher than what Sallinen et al (45) found during shorter night shifts. Ingre et al (46) found even higher levels of severe sleepiness (82\%) during early-morning work (approximately 12-hour shift) among train drivers. Son et al (51) also investigated if severe sleepiness 
increased across the sequence of 5-7 extended shifts in a row. The pattern across the week did not show any clear signs of accumulation. For example, for the 12hour night shift week, the peak $(66 \%)$ was observed on Monday, which was the first shift in the sequence, whereas the prevalence was somewhat lower $(60 \%)$ on Friday night.

One study measured physiological (EEG-defined) sleep in connection with extended (10-hour) shifts among underground mine workers (52). The sleep after the night shift was slightly $>5$ hours (291 minutes), which was approximately 40 minutes shorter than the sleep period prior to a day shift.

There are quite a few intervention studies on 12-hour shift systems and their effects on sleep and sleepiness, but we identified only one study that contained a control group. Lowden et al (53) used daytime workers at the same worksite as controls and showed that a 12-hour shift system was beneficial to sleep and sleepiness, although it should be emphasized that the 8-hour shift system included some shift combinations (eg, quick returns) that probably had a negative effect on sleep and sleepiness.

The other intervention studies have used pre/postchange designs without a control group and the results were quite inconsistent. Several studies by Rosa and colleagues (54-56) found that a change to 12-hour shifts resulted in higher sleepiness, in particular towards the end of the night shift, and slightly less sleep. However, the shift systems also differed with respect to shift changeover times, which might have influenced the results; it is difficult to estimate whether the obtained differences are related to shift duration or other characteristics of the shift system.

Some intervention studies using a pre/post-change design without a control group showed slight improvements for 12-hour shift systems. Using a questionnaire study, Duchon et al (57) compared a slowly rotating 8-hour shift system with a 12-hour one among a group of mine workers and found mainly improvements or no change in sleep duration and sleepiness for the 12-hour shifts. Williamson et al (58) compared a regular 12hour shift system with sequences of two 12-hour day shifts, followed by two 12-hour night shifts and then four days off, with a highly irregular shift system that was dominated by 8 -hour shifts ( $2-5$ consecutive work days) among a group of computer workers. The 12-hour shift system resulted in longer sleep duration on rest days and for those working the day shift, whereas sleep length was reduced among night shift workers. Tiredness during the shift (which may be interpreted as a proxy for sleepiness) was clearly improved in the 12-hour shift system. A more recent intervention study by Mitchell \& Williamson (59) showed very similar results among a group of employees at an electrical power station (ie, sleep and sleepiness did not deteriorate for the 12-hour shift system). Paley et al
(60) conducted an intervention study among firefighters comparing the change from a rotating 8-hour shift system to (i) a 2-shift system with 10-hour days and (ii) a 14hour night shift. The 2-shift system with extended shifts neither decreased sleep length nor increased sleepiness. An intervention study among miners by Hossain et al (52) evaluated the change from a backward-rotating 8hour shift system to a forward-rotating 10-hour system. The latter did not have any negative effects on sleep and sleepiness, although the lack of work during the early morning hours (03.00-7.00 hours) in the 10-hour shift system probably confounded the comparison.

Thus, most of the studies have suggested that 12hour shifts do not disturb sleep and increase sleepiness any more than regular 8 -hour 3 -shift systems. However, it may be that the former increase sleepiness, at least among certain occupations with a high mental work load, inadequate staff resources, insufficient rest breaks, or extended commuting time (61). It is also possible that the effects of 12-hour shifts may be exacerbated by other work hour characteristics, such as spells with many consecutive work days.

\section{Studies on permanent night work}

In the debate on how to organize work at night, some experts have claimed that permanent night work should be advantageous to sleep and wakefulness because it minimizes disruption of the master circadian clock (62). Relatively few field studies on permanent night work and its effects on sleep and sleepiness have been published during the last decade.

There are some meta-analyses showing that permanent night workers sleep more during the daytime (between night shifts) than rotating night workers $(18,57)$. For example, in an almost 20 -year-old metaanalysis, Wilkinson (62) concluded that permanent night workers obtained $\approx 1$ hour more sleep than workers on a rapidly rotating shift system. In a more recent meta-analysis, Pilcher and colleagues (18) estimated the average (daytime) sleep length of permanent night workers to be 6.6 hours, which was slightly longer than that of workers in rapidly rotating shift systems ( $\approx 6.5$ hours). Nevertheless, permanent day and evening workers clearly obtain more sleep ( $>7$ hours). Thus, permanent night workers are not likely to obtain more sleep than regular 3-shift workers when one takes the whole shift cycle into account (ie, also morning and evening shifts and days off), a suggestion also made by Folkard (63).

We are not aware of any intervention studies on permanent night work, but there are quite many observational studies that have compared permanent night workers with rotating shift workers. Barton and 
coworkers (64-66) developed a series of questionnaire studies showing that permanent night nurses report less sleep disturbances than rotating 3-shift workers. In their questionnaire study, Chan et al (67) also demonstrated that, compared to rotating night work, permanent night work is not associated with shorter sleep. In fact, complaints about experiencing poor sleep were higher among the rotators (39\%) than permanent night workers (28\%). Studies using physiological (EEG-defined) sleep recordings are rare, but Dahlgren (68) showed that permanent night workers had slightly better sleep (a lower amount of wake time after the onset of sleep) than rotating shift workers, although sleep length was still quite short (5.5 hours) and only marginally longer (10-15 minutes). A more recent study used actigraphs and compared permanent night workers with their day and evening counterparts (69). The results showed that permanent night work had the shortest sleep length (4.7 hours compared to 5.7 and 6.1 hours for the day and evening shifts, respectively) and reported poorer sleep quality than the other shift groups.

There are also studies showing that permanent night workers might be no worse off or even less sleepy during the night than rotating night workers (68-71). The studies should be interpreted with some caution since the sample sizes were often small and the comparisons were mostly made with slowly rotating 3 -shift work (which may not be the optimum way of organizing rotating night work). The methods for measuring sleepiness are not well established in some studies and the results might be confounded with other differences in work hours (eg, different start and finish times between the rotators and the permanent night workers).

Recently, Folkard (72) summarized six studies related to circadian adaptation to permanent night work and calculated that only a small group of workers $(<3 \%)$ seem to show complete circadian adaptation. Thus, it is likely that the extended sleep sometimes associated with permanent night work is not related to complete circadian adaptation; a later circadian phase in permanent night workers, or a minor circadian adjustment, might explain the small increase (30-60 minutes) during daytime sleep. This is in line with the studies showing an association between permanent night work and having a preference for evenings (ie, "eveningness") $(73,74)$. Permanent night workers are in many cases a highly select group, which includes individuals who have chosen such a shift system because they prefer to work at night (65). The selection might influence sleep behavior, for example, permanent night workers pay more attention to sleep.

In summary, the difference in the duration of daytime sleep and nighttime sleepiness between permanent and rotating night workers seems to be small. In addition, most of the studies were observational, which limits our ability to draw conclusions. Thus, no clear evidence exists showing that permanent night work is more advantageous than the rotating system. However, permanent night work may be a good solution for individuals who have a late circadian phase and the willingness and possibility to live in a night-oriented rhythm also during days off.

\section{Studies on shift systems during extended operations}

During the last decade, several studies have investigated extreme shift systems, for example in offshore operations and among medical interns who have on-call work during the night. Most of the studies have had an observational design and used sleep diaries.

There have been several studies on oil-rig workers, who have extended operations with 2 weeks offshore and 12-hour shifts, for example 14 days of night work or 7-day plus 7-night shifts. One would expect that sleep problems and sleepiness would accumulate across such a shift system. However, the results showed the opposite pattern, suggesting that the workers adapt to the longer spells of night work (75). Thus, sleep and sleepiness problems normally peak during the first day on the oil rig $(75,76)$. Towards the end of the week (or after 14 days of night work), bedtimes have been delayed and sleep length is often slightly $>7$ hours (even when measured with wrist activity monitoring). In fact, those working seven consecutive day shifts (starting at 06.00 hours) obtained less sleep (mean sleep was $\approx 6$ hours) and had similar sleepiness levels as those working during the night (62). Studies from Arendt's group (77) have also observed the relatively long sleep after night work during offshore operations. A drawback with the scheduling system that involves 14 consecutive night shifts is the increase in sleep-wake problems experienced by workers on their return home.

Clearly the results differ from those found in the studies on more conventional shift work. The explanation for the differences is that the oil-rig environment provides very good conditions for night work (76). The absence of social demands probably means that it is easier to give priority to sleep. The light exposure (eg, low levels of outdoor light after the night shift) associated with living offshore might also facilitate circadian adaptation to night work. On the other hand, a recent study suggested that a relatively large group of shift workers at an oil rig have problems with the extended shift systems and $23 \%$ of the shift workers were classified as suffering from shift work disorder (78).

Many consecutive, long ( $>12$ hours) work shifts will result in long working weeks. It has been shown in prospective epidemiological studies, including only daytime workers, that $>55$ hours of work per week are associated 
with shorter sleep length (79). In the healthcare sector, it is common to have both extremely long work shifts and long working weeks. A cross-sectional questionnaire study among nurses showed that shifts $>12.5$ hours increased the prevalence of nodding off incidents at work ( $4 \%$ versus $1 \%$ for shifts $<8.5$ hours) (80).

There have also been some recent intervention studies on work hours in healthcare. However, none of these studies used a control group. Lockely et al (81) carried out an intervention study in which interns working $>80$ hours (involving 24-hour shifts) during the traditional schedule restricted their maximum shift length to 16 hours. The intervention resulted in a reduction of weekly working hours to approximately 65 hours. The average sleep length per day after the intervention increased from 6.6 to 7.4 hours. Physiological (EEG-defined) sleepiness during on-call nights was reduced by approximately $50 \%$ following the intervention. The intervention also decreased the number of serious medical errors by approximately $30 \%$ in intensive care units (82). In a parallel study, the same group showed that extended work shifts ( $>24$ hours) were associated with an increased risk for being involved in a motor vehicle crash [odds ratio (OR) $2.3,95 \%$ confidence interval (95\% CI) 1.6-3.3] (83); one may assume that a large part of the crashes were related to sleepliness while driving. Another intervention study among junior doctors showed that a reduction of weekly work hours from 56 to 48 hours resulted in a significant $(33 \%)$ reduction in medical errors and a tendency towards more sleep (mean daily sleep length: 7.3 versus 6.8 hours for the 56-hour schedule, $\mathrm{P}=0.09$ ) (84). Thus, the latter studies clearly showed that a very long work week (>80 hours), which includes night work, is a safety risk and should be avoided.

To summarize, the studies on shift work during extended operations show somewhat inconsistent results. Although extended work hours during offshore operations show low levels of sleepiness and insufficient sleep, these results cannot be generalized to other industries and settings. For example, studies in healthcare settings have shown that long work shifts ( $>12$ hours) and weekly working hours ( $>48$ hours) result in a pronounced increase in severe sleepiness, which can lead to adverse safety consequences. A few intervention studies have shown that the negative impact on safety could be ameliorated by reducing weekly working hours and avoiding extended work shifts.

\section{Discussion}

In this review, our main aim was to examine the level of research evidence available on recommendations for how shift schedules should be designed in order to protect workers from sleep-wake disturbances. We examined this issue separately for various shift systems. An up-to-date overview of the topic could help in the development of more sleep- and alertness-friendly shift system scheduling; it would also help examine pertinent questions that are still open.

\section{General findings}

One of our most crucial observations was the shortage of controlled intervention studies. In fact, there were a reasonable number of intervention studies only for the category of regular 3 -shift systems. There were no intervention studies for the categories of either irregular shift systems or permanent night work, while the intervention studies on extended operations had no control group. Also, only one of the intervention studies, in which an 8 -hour shift system was replaced with a 12 -hour system, included a control group (53). We did not find any randomized controlled intervention studies for any of the shift system categories.

It is clear that the low number of controlled intervention studies make it difficult to present ergonomic recommendations for shift scheduling. Only controlled intervention studies can provide reliable evidence on the effects of shift rescheduling and give a concrete solution model for reducing the problem of a disturbed sleep-wake pattern in a particular shift system. With observational studies, one can identify the most problematic shift characteristics and then only indirectly infer which of the possible rescheduling options will result in marked positive changes in sleep and sleepiness.

The methods and designs used for measuring sleep and sleepiness were another limitation of the studies included in our review. The first point concerns the nature of the outcome measures. In most of the studies, sleep and sleepiness were measured only by subjective scales and questionnaires, and objective measures (such as actigraphic or polysomnographic recordings) were employed in only a few studies (eg, 22-24, 28, 31). Because subjective measures are often influenced by motivational factors and do not always match with physiological measures, it is possible that significant changes - associated with, for example, shift rescheduling - remain undetected. The second point concerns the way the measurements were conducted. In many studies, sleep and sleepiness were addressed retrospectively by means of questionnaires completed by participants only once or twice. In addition, sleep and sleepiness were not always the focus of shiftspecific questions (eg, "How do you sleep between night shifts?") but rather general questions about sleep and sleepiness over a certain period of time. Also, recovery of the normal sleep-wake rhythm and alertness between two consecutive spells of shifts were only rarely examined, but most of the studies were limited to a spell of shifts or 
periods preceding certain shifts. The importance of this limitation has been emphasized by recent experimental studies in which participants were repeatedly subjected to a period of short-term partial sleep restriction. Banks et al (85) and McCauley et al (86) showed that a single recovery night of extended sleep after cumulative sleep restriction leaves individuals' vulnerability to sleep loss at an elevated level. Rupp et al (87) found, in turn, that extension of sleep duration for several days before sleep restriction improved the rate of recovery. Both of these findings suggest that the ratio between the degree of accumulated sleep loss over a shift cycle and the amount of recovery sleep gained before the next shift cycle is important. Future studies should examine whether some shift systems really augment workers' vulnerability to sleep loss because of insufficient opportunities for recovery and what are the long-term consequences of working such shift systems for occupational safety and health.

It is evident that all the above-mentioned methodological solutions weaken the possibility of having reliable and valid results with respect to sleep and sleepiness in connection with different shifts, their combinations, and consecutive shift cycles. In all, methodological integrity and uniformity would enable a better comparision of the different shift systems and their characteristics and thus also provide a more solid base for guidance on which shift systems to implement in workplaces.

\section{Findings of the observational studies}

A main finding of the observational studies was that, irrespective of the shift system, night and early-morning shifts and quick returns are associated with short sleep and increases in sleepiness. The same pattern holds true for extended shifts ( $>16$ hours) and extremely long weekly working hours (>55 hours) $(79,80,81,84)$. These are not new findings and have been reported in previous reviews (eg, 13-15). Regarding the recommendations on the design of shift schedules, these findings reveal the common bottlenecks of various shift systems without providing any concrete remedy for the problem, as mentioned earlier. With this level of evidence, one can only recommend which main shift characteristics should be avoided, but not suggest working alternatives. In this kind of situation, the bottlenecks of a shift system can easily remain unsolved or be removed but at the expense of creating new problems.

Another important finding was that none of the observational studies compared different shift systems in terms of sleep and sleepiness, except those comparing permanent night work and rotating shifts $(18,57$, 62). The lack of this kind of observational study makes it difficult to recommend a regular shift system over an irregular one, even if intuitively the former seems be a better choice.
In terms of shift scheduling, perhaps the most valuable results of the observational studies were those that deal with shift start times and the duration of a break between two consecutive shifts. The findings demonstrate the limits for these shift characteristics beyond which sleep and alertness start to become significantly impaired. Examples of solutions that are likely to lead to unwanted consequences for sleep and alertness include starting a morning shift at 06.00 hours or earlier (38) and having a break of $<12$ hours between two consecutive shifts (37). On the other hand, the quality of this kind of knowledge is somewhat limited in shift scheduling, as a shift schedule can only rarely be developed by changing a single shift characteristic. This rule of thumb is based on the fact that a change in one characteristic often leads to changes in other characteristics as well (eg, a change in the start time of the morning shift often leads to a change in the finishing time of the night shift).

\section{Findings of the intervention studies}

We found the strongest evidence for ergonomic recommendations on improving shift schedules in studies on the regular 3-shift system. In particular, a change from slowly backward-rotating to rapidly forward-rotating shifts seems to be advantageous for alertness and, to some degree, sleep quality and the prevalence of napping $(31,32)$. These findings are, however, difficult to interpret, since the change in the direction of shift rotation was not the only change that occurred; for example, the number of consecutive shifts (speed of rotation) and the presence of quick returns were also modified. Given that shift work disturbs sleep and alertness by causing a misalignment between the sleep-wake schedule and the circadian timing system, it is possible that the change in the speed of shift rotation actually played a significant role in these interventions. It is likely that speeding up the rotation as such reduced disturbances in the sleep-wake pattern. In general, the issue of multiple simultaneous shift changes in intervention studies often makes it difficult to identify the most crucial modification or changes for improving sleep and sleepiness.

Some evidence was found for the argument that a change from an 8- to 12-hour shift system does not result in increases in sleep problems or in sleepiness (eg, 53, 57, 59). However, only one of these studies had a control group (53), which degrades the level of research evidence. Secondly and similarly to the studies on rescheduling regular 3-shift systems, the studies on the replacement of a 8-hour shift system with a 12-hour one often incorporated multiple shift changes, which makes it impossible to attribute the observed impacts solely to the manipulation of shift length.

Even if shift rescheduling was found to result in improvements in the sleep-wake pattern in some studies, 
the magnitude of the improvements was often quite limited. After rescheduling, workers usually gain around 30 minutes of extra sleep in association with, for example, the morning shift (less than $10 \%$ of total sleep time) and were still significantly sleepier than during day shifts (eg, 26, 28, 31). These results suggest that changing a certain shift characteristic or the existing shift system to another is not a "silver bullet" solution to mitigate sleep-wake problems in association with early-morning and night shifts.

One can assume that shift schedules that best support workers' sleep and alertness also lower the risk for shift work-related disorders and diseases in the long run. To the best of our knowledge, so far only the study of Viitasalo et al (32) has addressed this issue. The authors found that a change from a backward-rotating shift system to a rapidly forward-rotating one decreased sleepiness, but did not influence cardiovascular disease risk factors. However, a change from the backward-rotating shift system to a similar but more flexible shift system resulted in a decrease in systolic blood pressure and a declining trend in heart rate, even though no improvements in sleep or sleepiness occurred. These results suggest that a change in shifts can lead to improvements in sleepiness without improvements in health and vice versa. However, these results are preliminary due to the study's short follow-up time (7-8 months post-intervention) and small study groups $(\mathrm{N}=22-40)$. Larger studies with intensive field measurements, including biological markers of sleep, sleepiness, and health are required before conclusions can be drawn. In addition, the comprehensive knowledge of individuals' exposure to different shift systems is of utmost importance in this context.

To conclude, the level of research evidence for ergonomic recommendations on improving shift schedules is degraded by the lack of controlled intervention studies and observational studies comparing different shift systems and additionally the poor methodological integrity of studies. At the moment, the level of research evidence makes it possible to tell what individual shift characteristics and combinations should be avoided, but less can be said about working alternatives for shift schedules that markedly disturb sleep and alertness.

In the future, there are still several basic questions on shift scheduling that need to be answered. For instance, we do not know how many consecutive shifts of each type one can endure without accumulating sleepiness or the optimal way to distribute days off over a certain period of time to support recovery. In addition, the relationship of shift workers' sleep and sleepiness to their health in different shift systems and the benefits from individually scheduled shifts are amongst the issues that warrant extensive research in the future.

\section{References}

1. Schultes B, Schmid S, Peters A, Born J, Fehm HL. Sleep loss and the development of diabetes: a review of current evidence. Exp Clin Endocrinol Diabetes. 2005;113(10):563-67.

2. Spiegel K, Leproult R, Van Cauter E. Impact of sleep debt on metabolic and endocrine function. Lancet. 1999;354(9188):1435-39.

3. Copinschi G. Metabolic and endocrine effects of sleep deprivation. Essent Psychopharmacol. 2005;6(6):341-7.

4. Van Cauter E, Spiegel K, Tasalia E, Leproulta R. Metabolic consequences of sleep and sleep loss. Sleep Med. 2008;9 suppl 1:S23-S28.

5. Meier-Ewert HK, Ridker PM, Rifai N, Regan MM, Price $\mathrm{NJ}$, et al. Effect of sleep loss on C-reactive protein, an inflammatory marker of cardiovascular risk. J Am Coll Cardiol. 2004;43(4):678-83.

6. Van Leeuwen WMA, Lehto M. Karisola P, Lindholm H, Luukkonen R, Sallinen M, et al. Sleep restriction increases the risk of developing cardiovascular diseases by augmenting proinflammatory responses through IL-17 and CRP. Plos ONE. 2009;4(2):e4589.

7. Ayas NT, White DP, Manson JE, Stampfer MJ, Speizer FE, Malhotra A, et al. A prospective study of sleep duration and coronary heart disease in women. Arch Intern Med. 2003;27(3);205-9.

8. Goel N, Rao H, Durmer JS, Dinges DF. Neurocognitive consequences of sleep deprivation. Semin Neurol. 2009;29(4):320-39.

9. Sallinen M, Holm A, Hiltunen J, Hirvonen K, Härmä M, Koskelo J, et al. Recovery of cognitive performance from sleep debt: do a short rest pause and a single recovery night help? Chronobiol Int. 2008;25(2):279-96.

10. Van Dongen HPA, Maislin G, Mullington JM, Dinges DF. The cumulative cost of additional wakefulness: dose-response effects on neurobehavioral functions and sleep physiology from chronic sleep restriction and total sleep deprivation. Sleep. 2003;26(2):117-26.

11. Philip P, Åkerstedt T. Transport and industrial safety, how are they affected by sleepiness and sleep restriction? Sleep Med Rev. 2006;10:347-56

12. Åkerstedt T, Frelund M, Gillberg M, Jansson B. A prospective study of fatal occupational accidents - relationship to sleeping difficulties and occupational factors. J Sleep Res. 2002;11:6971.

13. Åkerstedt T. Sleepiness as a consequence of shift work. Sleep. 1988;11(1):17-34

14. Åkerstedt T. Shift work and disturbed sleep/wakefulness. Sleep Med Rev. 1998;2(2):117-128.

15. Åkerstedt T. Shift work and disturbed sleep/wakefulness. Occup Med. 2003:53(2);89-94.

16. Härmä M, Sallinen M, Ranta R, Mutanen P, Müller K. The effect of an irregular shift system on sleepiness at work in train drivers and railway traffic controllers. J Sleep Res. 2002;11(2):141-51. 
17. Knauth P, Hornberger S. Preventive and compensatory measures for shift workers. Occup Med. 2003;53(2):109-16.

18. Pilcher JJ, Lambert BJ, Huffcutt AI. Differential effects of permanent and rotating shifts on self-reported sleep length: a meta-analytic review. Sleep. 2000;23(2):155-63.

19. Timothy R, Driscoll TR, Grunstein RR, Rogers NL. A systematic review of the neurobehavioural and physiological effects of shiftwork systems. Sleep Med Rev. 2007;11:17994

20. Bambra CL, Whitehead MM, Sowden AJ, Akers J, Petticrew MP. Shifting schedules: the health effects of reorganizing shift work. Am J Prev Med. 2008;34(5):427-34.

21. Smith L, Folkard S, Tucker P, Macdonald I. Work shift duration: a review comparing eight and 12 hour shift systems. Occup Environ Med. 1998:55(4);217-29.

22. Tilley AJ, Wilkinson RT, Warren PS, Watson B, Drud M. The sleep and performance of shift workers. Hum Factors. 1982;24(6):629-41.

23. Äkerstedt T, Kecklund G, Knutsson A. Manifest sleepiness and the spectral content of the EEG during shift work. Sleep. 1991;14(3):221-5.

24. Torsvall L, Åkerstedt T, Gillander K, Knutsson A. Sleep on the night shift: 24-hour EEG monitoring of spontaneous sleep/ wake behavior. Psychophysiology. 1989;26(3):352-8.

25. Cruz C, Rocco PD, Hackworth C. Effects of quick rotating shift schedules on the health and adjustment of air traffic controllers. Aviat Space Environ Med. 2000;71(4):400-7.

26. Tucker P, Smith L, Macdonald I, Folkard S. Effects of direction of rotation in continuous and discontinuous 8 hour shift systems. Occup Environ Med. 2000;57(10):678-84.

27. van Amelsvoort LGPM, Jansen NWH, Swaen GMH, van den Brandt PA, Kant I. Direction of shift rotation among three-shift workers in relation to psychological health and work-family conflict. Scand J Work Environ Health. 2004;30(2):149-56.

28. Rosa RR, Härmä M, Pulli K, Mulder M, Näsman O. Rescheduling a three shift system at a steel rolling mill: effects of a one hour delay of shift starting times on sleep and alertness in younger and older workers. Occup Environ Med. 1996;53(10):677-85.

29. Barton J, Folkard S, Smith L, Poole CJ. Effects on health of a change from a delaying to an advancing shift system. Occup Environ Med. 1994;51(11):749-55.

30. Knauth P, Hornberger S. Changes from a weekly backward to quicker forward rotating shift systems in the steel industry. Int J Ind Ergon. 1998;21(3):267-73.

31. Härmä M, Tarja H, Irja K, Mikael S, Jussi V, Anne B, et al. A controlled intervention study of the effects of a very rapidly forward rotating shift system on sleep-wakefulness and well-being among young and elderly shift workers. Int J Psychophysiol. 2006;59(1):70-9.

32. Viitasalo K, Kuosma E, Laitinen J, Härmä M. Effects of shift rotation and the flexibility of a shift system on daytime alertness and cardiovascular risk factors. Scand J Work Environ Health. 2008;34(3):198-205.
33. Karlson B, Eek F, Orbaek P, Osterberg K. Effects of sleeprelated problems and self reported health after a change of shift schedule. J Occup Health Psychol. 2009;14(2):97-109.

34. Åkerstedt T, Gillberg M. Sleep disturbances and shiftwork. In: Reinberg A, Vieux N, Andlauer P, editors. Night and shift work: biological and social aspects: proceedings of the Fifth International Symposium on Night and Shift Work: Scientific Committee on Shift Work of the Permanent Commission and International Association on Occupational Health (PCIAOH) Rouen, 12-16 May 1980. Oxford: Pergamon Press; 1981. p 127-37.

35. Aguirre A, Foret J. Irregularity of working hours in railway workers and types of complaints. Int Arch Occup Environ Health. 1994;65:367-371.

36. Pilcher JJ, Coplen MK. Work/rest cycles in railroad operations: effects of shorter than 24-h shift work schedules and on-call schedules on sleep. Ergonomics. 2000;43(5):573-88.

37. Roach GD, Reid KJ, Sawson D. The amount of sleep obtained by locomotive engineers: effects of break duration and time of break onset. Occup Environ Med. 2003:60(12):e17.

38. Ingre M, Kecklund G, Åkerstedt T, Söderström M, Kecklund L. Sleep length as a function of morning shift-start time in irregular shift schedules for train drivers: self-rated health and individual differences. Chronobiol Int. 2008:25(2);349-58.

39. Torsvall L, Åkerstedt T, Gillberg M. Age, sleep and irregular work hours: a field study with electoencephalographic recording, catecholamine excretion and self-ratings. Scand J Work Environ Health. 1981:7;196-203.

40. Foret J, Lantin G. The sleep of train drivers: an example of the effects of irregular work schedules on sleep. In: Aspects of human efficiency. diurnal rhythm and loss of sleep. In: Colquhoun WP, editor. London: The English Universities Press Ltd; 1972. p 273-82.

41. Kecklund G, Åkerstdt T, Lowden A. Morning work: effects of early rising on sleep and alertness. Sleep. 1997;20(3):21523.

42. Torsvall L, Akerstedt T. Sleepiness on the job: continuously measured EEG changes in train drivers. Electroenceph Clin Neurophysiol. 1987:66(6);502-11.

43. Mitler MM, Miller JC, Lipsitz JJ, Walsh JK, Wylie CD. The sleep of long-haul truck drivers. N Engl J Med. 1997;337(337):755-61.

44. Sallinen M, Härmä M, Mutanen P, Ranta R, Virkkala J, Müller K. Sleep-wake rhythm in an irregular shift system. J Sleep Res. 2003;12(2):103-12.

45. Sallinen M, Härmä M, Mutanen $P$, Ranta R, Virkkala J, Müller $\mathrm{K}$. Sleepiness in various shift combinations of irregular shift systems. Ind Health. 2005;43(1):114-22.

46. Ingre M, Keclund $\mathrm{G}$, Åkerstedt T, Kecklund L. Variation in sleepiness during early morning shifts: a mixed model approach to an experimental field study of train drivers. Chronobiol Int. 2004;21(6):973-90.

47. Tucker P, Smith L, Macdonald I, Folkard S. The impact of early and late shift changeovers on sleep, health, and wellbeing in 8-and 12-hour shift systems. J Occup Health Psychol. 1998;3(3):267-75. 
48. Tucker P, Smith L, Macdonald I, Folkard S. Shift length as a determinant of retrospective on-shift alertness. Scand J Work Environ Health. 1998;24 suppl 3:49-54.

49. Tucker P, Smith L, Macdonald I, Folkard S. Distribution of rest days in 12 hour shift systems: impacts on health, wellbeing, and on shift alertness. Occup Environ Health. 1999;56(3):206-14.

50. Bednick LD, Lerman SE, Baker TL, Jones H, Czeisler CA. Sleep and alertness in a 12-hour rotating shift work environment. J Occup Med. 1994;36(12):1295-300.

51. Son M, Kong J-O, Koh S-B, Kim J, Härmä M. Effects of long working hours and the night shift on severe sleepiness among workers with 12-hour shift systems for 5 to 7 consecutive days in the automobile factories of Korea. J Sleep Res. 2008;17:385-94.

52. Hossain JL, Reinish LW, Heslegrave RJ, Hall GW, Kayumov L, Chung SA, et al. Subjective and objective evaluation of sleep and performance in daytime versus nighttime sleep in extended-hours shift-workers at an underground mine. J Occup Environ Med. 2004;46(3):212-26.

53. Lowden A, Kecklund G, Axelsson J, Åkerstedt T. Change from an 8-hour shift to a 12-hour shift, attitudes, sleep, sleepiness and performance. Scand J Work Environ Health. 1998:24 suppl $3 ; 69-75$.

54. Rosa RR, Colligan MJ, Lewis P. Extended work days: effects of 8-hour and 12-hour rotating shift schedules on performance, subjective alertness, sleep patterns, and psychosocial variables. Work Stress. 1989;3(1):21-32.

55. Rosa RR. Performance, alertness, and sleep after 3-5 years of 12 shifts: a follow study. Work Stress. 1991;5(2):107-16.

56. Rosa R, Bonnet M. Performance and alertness on 8-hour and 12-hour rotating shift schedules at a natural gas utility. Ergonomics. 1993:36(10);1177-93.

57. Duchon JC, Keran CM, Smith TJ. Extended workdays in an underground mine: a work performance analysis. Hum Factors. 1994;36(2):258-68.

58. Williamson A, Gower CGI, Clarke BC. Changing the hours of shiftwork: a comparison of 8- and 12-hour shift rosters in a group of computer operators. Ergonomics. 1994;37(2):287-98.

59. Mitchell RJ, Williamson AM. Evaluation of an 8 hour versus a 12 hour shift roster on employees at a power station. Appl Ergon. 2000;31:83-93.

60. Paley MJ, Price JM, Tepas DI. The impact of a change in rotating shift schedules: a comparison of the effects of 8,10 and $14 \mathrm{~h}$ work shifts. Intern J Ind Erg. 1998;21(3/4):293-305.

61. Rosa RR. Extended workshifts and excessive fatigue. J Sleep Res. 1995;4 suppl 2:51-6.

62. Smith MR, Fogg LF, Eastman CI. A compromise circadian phase position for permanent night work improves mood, fatigue, and performance. Sleep. 2009;32(11):1481-9.

62. Wilkinson RT. How fast should the night shift rotate? Ergonomics. 1992;35(12):1425-46.

63. Folkard S. Is there a "best compromise" shift system? Ergonomics. 1992;35(12):1453-63.

64. Barton J, Folkard S. The response of day and night nurses to their work schedule. J Occup Psychol. 1991;64:207-18.
65. Barton J, Smith L, Totterdell P, Spelten E, Folkard S. Does individual choice determine shift system acceptability? Ergonomics. 1993:36(1-3);93-9.

66. Barton J, Spelten E, Totterdell P, Smith L, Folkard S. Is there an optimum number of night shifts?: relationship between sleep, health and well-being. Work Stress. 1995;9(2/3):109-23.

67. Chan OY, Gan SL, Yeo MH. Study on the health of female workers on 12 hour shifts. Occup Med. 1993;43:143-8.

68. Dahlgren K. Adjustment of circadian rhythms and EEG sleep functions to day and night sleep among permanent nightworkers and rotating shiftworkers. Psychophysiology. 1981;18(4):381-91.

69. Burch JB, Yost M, Johnson W, Allen E. Melatonin, sleep, and shift work adaptation. J Occup Environ Med. 2005;47(9):893-901.

69. Patkai P, Åkerstedt T, Pettersson K. Field studies of shift work, I: temporal patterns in psychophysiological activation in permanent night workers. Ergonomics. 1977;20(6):611-9.

70. Wilkinson R, Allison S, Feney M, Kaminska Z. Alertness of night nurses: two shift systems compared. Ergonomics. 1989;32(3):281-92.

71. Totterdell P, Spelten E, Barton J, Smith L, Folkard S. On-shift and daily variations in self-report and performance measures in rotating-shift and permanent night nurses. Work Stress. 1995;9(2/3):187-97.

72. Folkard S. Do permanent night workers show circadian adjustment?: a review based on the endogenous melatonin rhythm. Chronobiol Int. 2008;25(2):215-24.

73. Petru R, Wittman M, Nowak D, Birkholz B, Angerer P. Effects of working permanent night shifts and two shifts on cognitive and psychomotor performance. Int Arch Occup Environ Health. 2005;78:109-16.

74. Burch JB, Tom J, Zhai, Y, Criswell L, Leo E, Ogoussan K. Shiftwork impacts and adaptation among health care workers. Occup Med (Lond). 2009;59:159-66.

75. Bjorvatn B, Kecklund $\mathrm{G}$, Åkerstedt T. Rapid adaptation to night work at an oil platform, but slow readaptation after returning home. J Occup Environ Med. 1998;40(7):601-8.

76. Bjorvatn B, Stangenes K, Øyane N, Forberg K, Lowden A, Holsten F, et al. Subjective and objective measures of adapation and readaptation to night work on an oil rig in the North Sea. Sleep. 2006;29(6):821-9.

77. Barnes RG, Forbes MJ, Arendt J. Shift type and season affect adaptation of the 6-sulphatoxymelatonin rhythm in offshore oil rig workers. Neurosci Lett. 1998;252(3):179-82.

78. Waage S, Moen BE, Pallesen S, Eriksen HR, Ursin H, Akerstedt T, et al. Shift work disorder among oil rig workers in the North Sea. Sleep. 2009;32(4):558-65.

79. Virtanen M, Ferrie JE, Gimeno D, Vahtera J, Elovanio M, Singh-Manoux A, et al. Long working hours and sleep disturbances: the Whitehall II prospective cohort study. Sleep. 2009;32(6):717-8.

80. Scott LD, Rogers AE, Hwang W-T, Zhang Y. Effects of critical care nurses' work hours on vigilance and patients. Am J Crit Care. 2006;15(1):30-7. 
81. Lockley SW, Cronin JW, Evans EE, Cade BE, Lee CJ, Landrigan CP, et al. Effects of reducing interns' weekly working hours on sleep and attentional failures. N Engl J Med. 2004;351(18):1829-37.

82. Landrigan CP, Rothschild JM, Cronin JW, Kaushal R, Burdick E, et al. Effects of reducing interns' work hours on serious medical errors in intensive care units. N Engl J Med. 2004;351(18):1838-48.

83. Barger LK, Cade BE, Ayas NT, Cronin JW, Rossner B, Speizer FE, et al. Extended work shifts and the risk of motor vehicle crashes among interns. N Engl J Med. 2005;352(2):125-34.

84. Cappucio FP, Bakewell A, Taggert FM, Ward G, Sullivan JP et al. Implementing a $48 \mathrm{~h}$ EWTD-compliant rota for junior doctors in the UK does not compromise patients' safety: assessor-blind pilot comparison. Q J Med. 2009;102:271-82.
85. Banks S, Van Dongen H, Dinges D. Response to sleep restriction depends upon pre-existing sleep debt. Sleep Abstract Supplement[30]. 2007:A119.

86. McCauley P, Kalchev V, Smith AD, Belenky G, Dinges DF, Van Dongen HPA. A new mathematical model for the homeostatic effects of sleep loss on neurobehavioral performance. J Theor. Biol. 2009;256(2):227-39.

87. Rupp TL, Wesensten NJ, Bliese PD, Balkin TJ. Banking sleep: Realization of benefits during subsequent sleep restriction and recovery. Sleep. 2009;32(3):311-21.

Received for publication: 3 November 2009 